\title{
A Quantitative Model for Option Sell-Side Trading with Stop-Loss Mechanism by Using Random Forest
}

\section{Chi-Fang Chao}

National Taipei University of Technology

Yu-Chen Wang

National Taipei University of Technology

Mu-En Wu ( $\square$ mnasia1@gmail.com )

National Taipei University of Technology https://orcid.org/0000-0002-4839-3849

\section{Research Article}

Keywords: Option, Trading Strategy, Money Management, Kelly Criterion, Machine Learning

Posted Date: August 6th, 2021

DOI: https://doi.org/10.21203/rs.3.rs-769898/v1

License: (c) This work is licensed under a Creative Commons Attribution 4.0 International License.

Read Full License 


\title{
A Quantitative Model for Option Sell-Side Trading with Stop-Loss Mechanism by Using Random Forest
}

\author{
Chi-Fang Chao · Yu-Chen Wang · Mu-En Wu*
}

Received: date / Accepted: date

\begin{abstract}
Due to the characteristics of high leverage and low margin, option is very suitable for quantitative trading by applying portfolio management to control the profit and risk. The money management is an important issue to build a portfolio especially for option sell-side trader, since the profit is only the premium, while the loss is unlimited. In this research, we propose a model for option sell-side strategy to estimate the win-rate of option by the premium, time to maturity, and volatility based on statistical approach and random forest algorithm. The prediction of the model is visualized through heatmap which can reveal the profitable trading range intuitively, we use the precision score to evaluate the performance in these two models and proof the effectiveness and robustness of predictive model proposed by random forest algorithm. In the future, we plan to apply other machine learning algorithm to propose the predictive model for spread trading.
\end{abstract}

Keywords Option · Trading Strategy · Money Management · Kelly Criterion · Machine Learning

هu-En $\mathrm{Wu}^{*}$ (Corresponding Author)

E-mail: mnwu@mail.ntut.edu.tw

Chi-Fang Chao

E-mail: s9860320@gmail.com

Yu-Chen Wang

E-mail: t108ab8009@ntut.org.tw

Department of Information and Finance Management, National Taipei University of Technology, Taipei City, Taiwan.

\section{Introduction}

1.1 Background and motivation

With the evolution of the financial market, investment tools and trading strategies have become increasingly diversified. Option is a common and popular derivative financial product, and investors often use it for hedging or speculation (Merton, 1973). However, option have the characteristics of high leverage which can earn higher profits with lower costs, and produce larger losses. Investors often need to bear higher risk (Yang et al., 2017). Especially when the option sell-side operates Naked Option, the maximum profit is only the premium, but the maximum loss is unlimited. When the market fluctuates sharply, it is easy to be called by the exchange for margin or forced liquidation (Cox et al., 1979; Liu et al., 2021). Therefore, fund management and risk control are particularly important for option sell-side.

In recent years, the financial market has been turbulent, and the importance of fund management has become a very importance research topic in both the industry and academia. For investors, proper fund management can effectively allocate available funds to various markets or financial products, and further adjust the size of the funds according to the risk and winrate of the transaction, in order to pursue long-term stable profits. The concept of money management originated from Kelly Criterion (Kelly Jr, 2011) proposed by John Larry Kelly in 1956. Which initially used to study the problem of incomplete message transmission due to communication noise, and then it was derived into a formula of the optimal betting ratio (Thorp, 2011; Stutzer, 2011; Wu et al., 2017). Then, in 2008, Edward O. Thorp applied the Kelly criterion to blackjack poker 
games, sports betting, and securities markets (Thorp, 2011). The Kelly criterion is applicable to traditional gambling games with an unlimited number of bets and a fixed win-rate and odds (Wu et al., 2015). In the case of a fixed profit-to-loss ratio, the expected value and the optimal betting ratio can be calculated, and repeated betting under the condition of a fixed ratio can maximize the asset growth (MacLean et al., 2011, 2010; Wu et al., 2017). However, there are still many differences between financial trading and traditional gambling, and it is difficult for real trading to achieve the unlimited betting. Moreover, the market is unpredictable, and the win-rate and odds of a trading cannot be completely accurately estimated (Wu et al., 2015). Therefore, if the Kelly criterion is to be applied to the financial market, it is necessary to formulate a perfect trading strategy. This strategy requires fixed odds and as accurately as possible the probability of winning. In a limited number of transactions, if the estimated win-rate of the strategy is almost equal to the actual profit ratio, the Kelly criterion can be applied to calculate the expected value and the optimal betting ratio.

From the above, there is a gap between the actual trading and the Kelly criterion applicable to traditional gambling. However, in many financial commodities. Option can know the maximum profit and maximum loss of the transaction through a combination strategy or a stop-loss method. And then control the transaction risk and profit (Wu and Chung, 2018; Wu and Hung, 2018). The option of fixing the stop-loss is similar to the way of the Kelly criterion to fix the odds, so if an accurate trading win-rate can be obtained. Then the Kelly criterion can be applied to the option strategy, and appropriate capital allocation and position control can be done (Bermin et al., 2019; Bermin and Holm, 2021). In addition to the fixed odds, the estimation of the win-rate will be an important factor. If there is an error between the predicted win-rate of the strategy and the actual profit probability, it will affect the long-term capital gains and losses. Therefore, the accuracy of win-rate estimation will be one of the important research topics in this study.

The win-rate is the primary consideration in the process of making a trading strategy. A high win-rate means more opportunities for profit. For option trading, the win-rate of option sell-side is much higher than that of option buyers (Evans et al., 2009). In view of this, this study will develop trading strategies from the perspective of option sell-side. Although the option sellside has a high win-rate, but the risk is relatively high. Its maximum profit is only the premium, but the maximum loss is unlimited. If there is a sharp rise or fall during the contract period, the option sell-side may suf- fer a huge loss. Therefore, the establishment of a stoploss mechanism is an important key to the development strategy of this study. If the trading strategy fixes the stop loss point that means we have the odds. Just use statistical methods or machine learning techniques to estimate an accurate win-rate (Ruf and Wang, 2020). The Kelly criterion mentioned above can be applied to make the best allocation of funds to achieve long-term stable returns.

\subsection{Purposes}

Since options have a combination strategy operation method, investors can fix their profits and losses (Brenner and Subrahmanyam, 1994). This study proposes a trading strategy for option sell-side with stop-loss mechanism. Apply the concept of fixed odds of the Kelly criterion to the strategy, and formulate a premium doubling stop-loss system to control the risks. Then, statistical methods and machine learning algorithms are used to estimate the win-rate (Nabipour et al., 2020; Jang et al., 2021). The model training feature value mainly uses the premium and the time to maturity and adds the volatility as a filter. Finally, based on the overall experimental results and the predicted win-rate of the model, a stable and profitable trading range was screened out, and the actual feasibility of the strategy was proved.

\section{Preliminaries}

\subsection{Characteristics of Option}

Option is a derivative financial product that investors often use to hedge or speculate. Option trading is ZeroSum Game, and buy-side profit (loss) is the sell-side loss (profit) (Brenner and Subrahmanyam, 1994). When the contract is established, the option buy-side has the right to buy or sell a certain amount of the subject matter at a certain price on a certain date in the future. In order to enjoy this right, buy-side must first pay premium to the sell-side, and sell-side is obliged to perform the content stipulated in the contract on the expiry date after receiving the premium. The option sell-side has obligations but no rights, so it is necessary to pay Margin first to avoid being unable to perform the contract.

The influencing factors of option value include two: Intrinsic Value and Time Value. Among them, Time Value is very important to the option sell-side, so we will further explain these two values. Intrinsic value used to determine whether the current price of the option has a fulfillment value (Wiggins, 1987). As shown 
in Table 1, suppose we buy a call with a strike price of 12,800. During the contract period, when the index happens to be at the strike price of 12800 , the profit from the current contract performance will be zero, which is called "At the Money". When the index is less than the strike price of 12800 , the current performance will cause a loss, which is called "Out of the Money". When the index is greater than the strike price of 12,800 , the spread and profit can be made when the contract is fulfilled, which is called "In the Money". Therefore, only the "In the Money" option have the intrinsic value.

Table 1 Comparison table of market index market and option sell-side profit and loss

\begin{tabular}{ccc} 
Call & Strike Price & Put \\
\hline Out of the Money & 12700 & In the Money \\
\hline At the Money & 12750 & At of the Money \\
\hline In of the Money & 12800 & \\
& 12850 & Out the Money
\end{tabular}

Time value is like the expectation that the option buy-side waits for the option to move from "Out of the Money" to "In the Money". The in-the-money option has intrinsic value that can make the buy-side profitable, while the out-of-the-money option has time value. When the value of time gradually declines, it is a moment in favor of the sell-side. It is worth noting that as the settlement date approaches, the time value of the out-of-the-money option will gradually fade and approach zero. Option sell-side can earn time value through the passage of time. When the option premium returns to zero during settlement, the sell-side makes a profit, as shown in Figure 1.

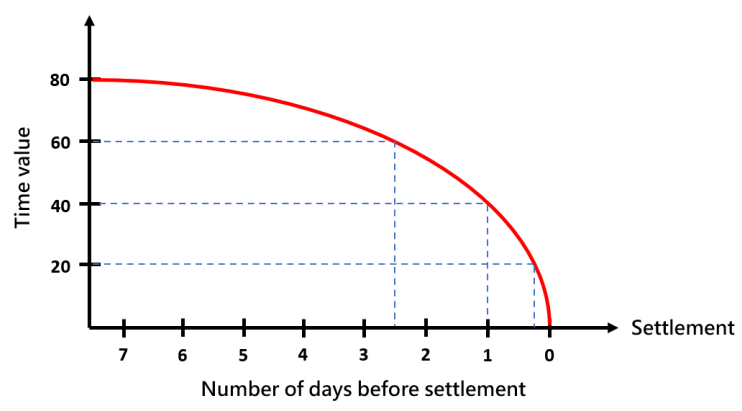

Fig. 1 Time value gradually decrease as the settlement approaches

\subsection{Random Forest Algorithm}

Random forest is a model training method of ensemble learning proposed by Leo Breiman, which can be regarded as an extension of decision tree (Breiman, 2001). As shown in Figure 2, the method is to randomly take samples from the data set using Bagging and form multiple decision trees (CART), and the result of each decision tree will form a class (Belgiu and Drăguţ, 2016; Pal, 2005). The principle of random forest is based on a decision tree as a basic classifier, combining the results of multiple decision trees, and using voting to select the category with the most votes among many decision trees(Pal, 2005). Random forest first uses Bagging to take samples from the data set and form several decision trees, and then combine many different decision trees to form a new learner. Compared with a simple decision tree, random forest has stronger generalization ability, can handle more input variables, and can evaluate the importance of each variable. For data sets with uneven classification, random forest can reduce the error and less likely to cause overfitting problems. In addition, random forest can also effectively deal with the problem of missing values. When there are many missing values in the data set, the classification accuracy can still be maintained through the evaluation method. Due to the outstanding performance of this algorithm, its application fields have spread to business analysis, financial data, and medical research, etc., and have made many contributions.

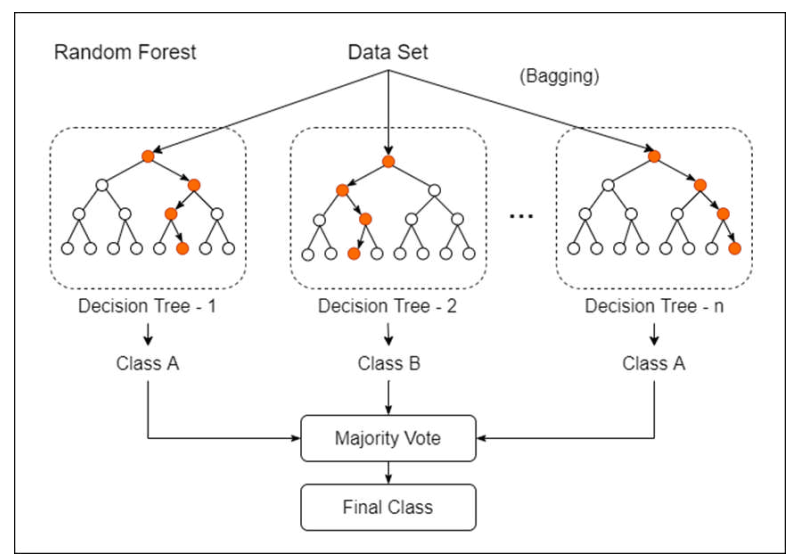

Fig. 2 Schematic for Random Forest Algorithm

\section{Methods}

In this section, we first introduce the concept of option sell-side strategy proposed in this paper. Then we esti- 
mate the win-rate with quantitative features by statistical approach and apply heatmap to visualize the winrate under with different features. We also propose the advanced win-rate predictive model based on random forest algorithm which is one of the popular machine learning algorithms and the additional quantitative feature, volatility has been considered in the model.

\subsection{Structure for sell-side trading strategy}

Due to the naked selling option has the characteristics of limited profit and unlimited loss, we propose a sellside strategy with stop-loss mechanism to constrain the maximum loss of sell-side option. In this strategy, we set up the $S L R$ and short the option, the position will be closed instantly to stop the loss if the premium of option rises to $(1+B)$ times after the trader sold the option, otherwise the position will be hold to expiration time, the flowchart of the strategy is shown in Figure 3.

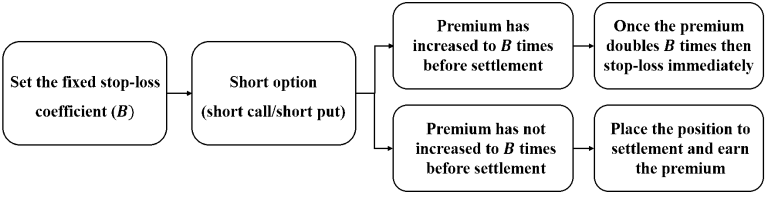

Fig. 3 The structure of option sell-side strategy with stop-loss mechanism

This strategy is composed of short call option and short put option and only focus on the contract which time to maturity is less than one week to ensure the efficiency of fund utilization. Time to maturity of option can be represented as $\left\{t_{1}, t_{2}, \ldots, t_{i}, \ldots, t_{s}\right\}$, which $t_{i}$ is the trade time, $t_{j}$ is the stop loss time and $t_{s}$ is the expiration time, as shown in Fig. 4. If the premium of option rises to $(1+B)$ times before $t_{s}$ that is if Call $_{k, t_{j}} \geq$ Call $_{k, t_{i}} \times(1+B)$ where $t_{i}<t_{j} \leq t_{s}$, the position should be closed to stop the loss or the position will be hold to $t_{s}$.

\begin{tabular}{ccc} 
trade time & stop-loss & settlement \\
\hline$t_{i}$ & $t_{j}$ & $t_{s}$ \\
& &
\end{tabular}

Week_Time $=\left\{t_{1}, t_{2}, \ldots, t_{i}, \ldots, t_{j}, \ldots, t_{S}\right\}$

Fig. 4 Schematic for Random Forest Algorithm
Take an example, a sell-side trader shorts a Call option with strike price of $\mathrm{K}$ for 20 at $t_{i}$ and $S L R$ of 1 $\left(\right.$ Call $\left._{k, t_{i}}=20, S L R=1\right)$, if the premium rises to stoploss point $40\left(\mathrm{Call}_{k, t_{i}} \times(1+B)\right)$ on $t_{j}$, the position should be closed, or the position will hold to expiration time $t_{s}$

There are three possible outcomes in our sell-side strategy, supposes a sell-side trader shorts a Call option with strike price of 10250 for 20 on Monday 10:00 A.M. and $S L R$ of 1, it can be represented as Short Call $_{10250, \text { Monday 10:00 A.M }}$ 1 , first outcome is if the premium has risen to 40 on $t_{j}$, the position should be closed, even if the option is out-of-money at $t_{s}$, the trader still loss 1,000 dollars $(50 \times 1 \times 20)$, as shown in Figure $5(\mathrm{a})$; second outcome is the premium of option has not risen to 40 on $t_{j}$, the position be hold to expiration and the trader can get 1,000 dollars since the out-of-money option does not have any exercise value, as shown in Figure 5(b); the last outcome is the premium has rise more than one times of premium on $t_{j}$ and keep rise until $t_{s}$, but the trader has closed the position at stop-loss point, which could control the maximum loss in 1 times of premium without the over loss, as shown in Figure 5(c). The description of the features are shown in Table 2 .

Since the maximum loss is controlled by $S L R$ times of premium and the maximum profit is the premium only, the odds of the trade have been fixed, we could estimate the win-lose ratio and measure whether the strategy with specific features is profitable by expected value calculated by odds and win-lose ratio.

\subsection{Win-loss ratio evaluation and visualization}

In this section, we use statistical approach to estimate the win-lose ratio in certain periods based on ODDS and quantitative features, including TTM and PI. Premium usually be closed to zero at expiration time, since the time value decayed accelerates as $t_{s}$ draw closer. Unless the Premium has risen to $(1+B)$ times, option sellside trader can get the full premium paid by buy-side trader when the Premium is zero, therefore, the measurement of win and lose of the trade is whether the premium of the option has risen to stop-loss point before expiration, the win-lose ratio in this section refers to the ratio of winning times to total times with specific TTM and PI, which could be defined by Equation 3.1.

win-lose $\operatorname{ratio}_{T T M=t, P I=p}=\frac{\mid\{\forall x \text { in dataset }: x>0\} \mid}{\mid\{\forall x \text { in dataset }\} \mid}$

In order to verify the feasibility and effectiveness of our strategy, the data has been separated into training set and testing set as the comparison and evaluate the 
Table 2 Description of the feature

\begin{tabular}{lll} 
Feature & Abbreviation & Description \\
\hline Time to maturity & $T T M$ & The remaining time of the option \\
Trade time & $t_{i}$ & The time that the trader shorts the option \\
Stop-loss time & $t_{j}$ & The time that the trader closes the option to stop the loss \\
Expiration time & $t_{s}$ & The time for option settlement to measure whether it has exercise value \\
\hline Premium & Premium & The premium of the option \\
Premium Interval & $P I$ & The premium interval of the option, each five points is a unit \\
\hline Stop-loss ratio & $S L R$ & The ratio is used to calculate the stop-loss point of premium \\
\hline
\end{tabular}

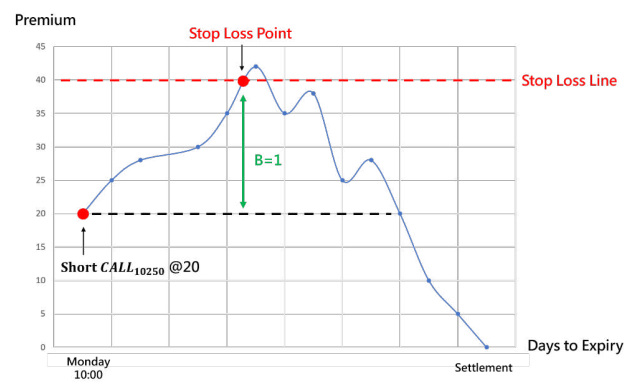

(a) The premium has risen to stop-loss point and closes to zero at expiration

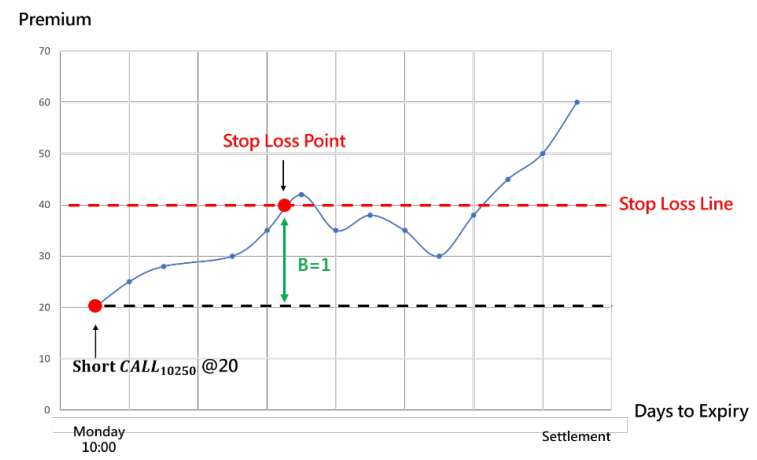

(b) The premium has not risen to stop-loss point and closes to zero at expiration

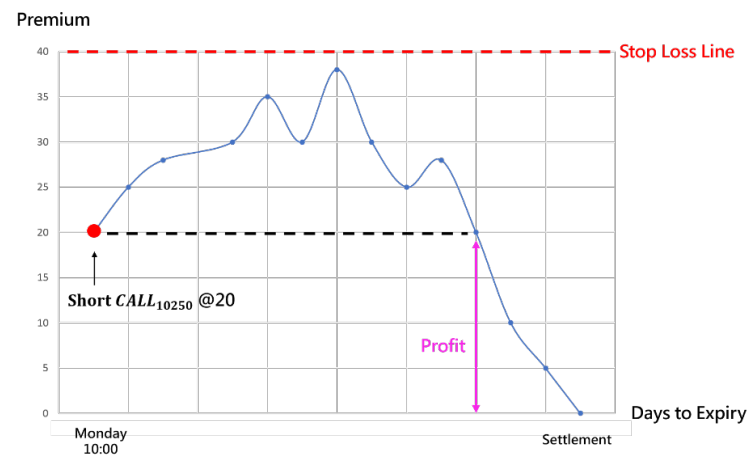

(c) The premium has risen to stop-loss point and above to stop-loss point at expiration

Fig. 5 Possible outcome of the sell-side strategy win-lose ratio with specific stop-loss ratio by statistical approach respectively, as shown in Figure 6. Take short call option for instance, the win-lose ratio can be expressed by Equation 2.

Prob. $\left\{\operatorname{Call}_{k, t_{j}}<(1+B) \times\right.$ Call $_{k, t_{i}}$ for all $\left.t_{i}<t_{j} \leq t_{s}\right\}(2)$

However, even if Equation 2 is satisfied, the sellside trader may still incur losses since $\mathrm{Call}_{k, t_{s}}$ might between 1 and $(+B)$ times of premium, but generally speaking, $\mathrm{Call}_{k, t_{s}}$ less then 5 in most of the time since the premium usually close to 0 while the option is outof-money at $t_{s}$.

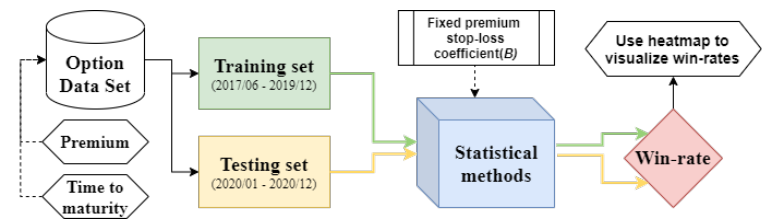

Fig. 6 Win-loss ratio estimation based on Premium and Time to Maturity

With the estimation of win-lose ratio, we utilize the heatmap to visualize the distribution of win-lose ratio. The vertical axis is $P I$ and the horizontal axis is $T T M$, which is shown in Figure 7. Each block in heatmap represents the win-lose ratio for strategy with specific features. Since the benchmark of the color is even winlose ratio inferred by fixed odds, the heatmap could reveal the profitable strategy through the intensity of the color.

3.3 Using Random Forest to predict win-rate for our strategy

Besides to TTM and $P I$, there still many quantitative features can be used to estimate the win-lose ratio of our strategy that whether the premium doubled before expiration time. However, the computation complexity will increase dramatically with the number of features while we use statistical approach, therefore, in order 


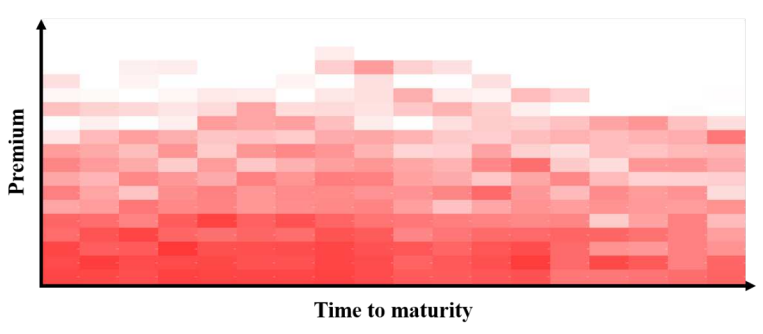

Fig. 7 Visualization of win-loss ratio with heatmap

to solve this issue, other estimating approach should be considered, such as machine learning, neural network and financial engineering etc., these approaches can take more features into consideration to estimate the win-rate without worrying about the penalty caused by computational complexity, the process shown in Figure 8 .

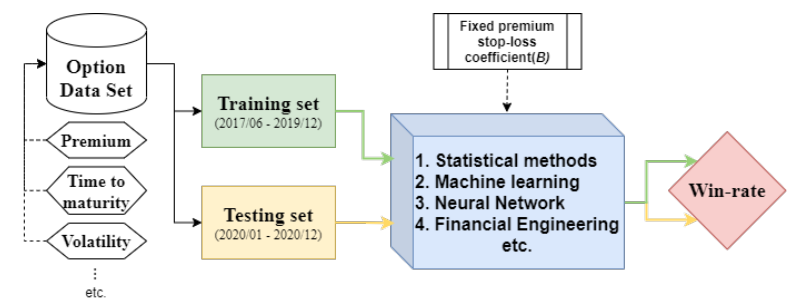

Fig. 8 Possible approaches to predict the win-rate with multiple features

Since we only focus on the option which $T T M$ is less than one week, the volatility of underlying asset has a significant impact on the premium of the option, therefore, we take volatility as the additional feature to analyze the influence of volatility on win-rate for the strategy. Furthermore, since the outcome of whether premium is doubled before expiration time only can be classified into two classes, such as doubled and nondoubled, which is the binary classification problem, therefore, we select random forest algorithm to propose the predictive model, which utilizes PI, TTM and volatility in different frequencies as features to predict whether the premium is doubled before expiration time, abbreviated as is_double.

To evaluate the performance of win-rate predictive model, the data has been divided into training set and testing set according to the time series. Random forest algorithm utilizes bootstrap aggregating techniques to randomly sample the data and features from set with replacement to generate numbers of decision tree to ensemble a strong classifier, the principle is shown in Figure 9. Since each data is classified in one class, we could predict the win-rate with specific features in training set and testing set respectively and make comparison to ensure the robustness of the predictive model. We also apply confusion matrix which is a commonly used index in machine learning algorithm to measure the consistency of predicted outcome and realized outcome.

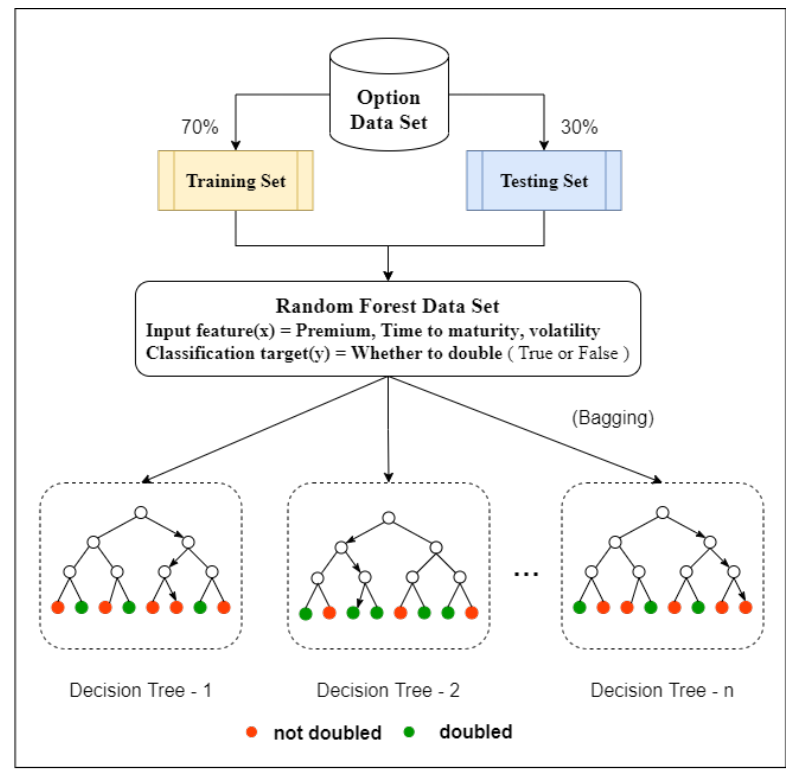

Fig. 9 Principles of Random Forest Algorithm

\section{Experiments}

This study used the historical data of Taiwan Stock Price Index Option (TXO) from June 2017 (after the implementation of after-hours trading started) to December 2020 for research. The trading frequency was in minutes, and the close price of per minute was set as the premium for the option. The call and put option were be analyzed separately, and the contract period is the weekly option (settle on every Wednesday). This chapter first used statistical methods to present the distribution of win-rate under fixed odds, and selected profitable trading ranges. Then, apply the random forest algorithm to focus on the profitable trading range for more in-depth win-rate estimation.

\subsection{Using statistical methods to calculate the win-rate}

This section reveals the win-lose ratio of statistical experiments. First, we divided the historical data into training set (data period from June 2017 to December 2019) and testing set (data period from January 2020 to December 2020). Then use $P I$ and TTM as two major 
features, and calculate the win-lose ratio under these two major features. The win-lose ratio in this study referred to the "probability that the premium has not doubled before expiry". Therefore, we first marked the data of "premium not doubled" in the data as 1 , and the data of "premium doubled" as 0 . After the win-lose ratio is calculated, heatmap is used to visualize the distribution result of the win-lose ratio, as shown in Figure 10 .

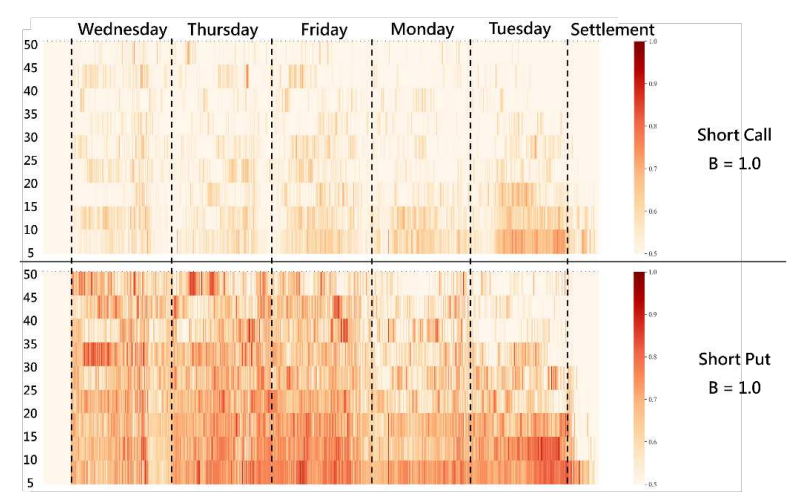

Fig. 10 Win-rate heatmap of statistical method $(S L R=1.0)$

The top and bottom half of Figure 10 is the heatmap of win-lose ratio for short call and short put respectively. The vertical axis of the heatmap is $P I$, from bottom to top is from 5 to 50 points; and the horizontal axis is the TTM, and the time is in minutes. The weekly option opens and closes at 08:45 and 13:30 every Wednesday. The total number of minutes in the trading period will be 10365 minutes. Therefore, the horizontal axis of the heatmap is 10365 (open) to 0 (close) from left to right, as shown in Figure 11.

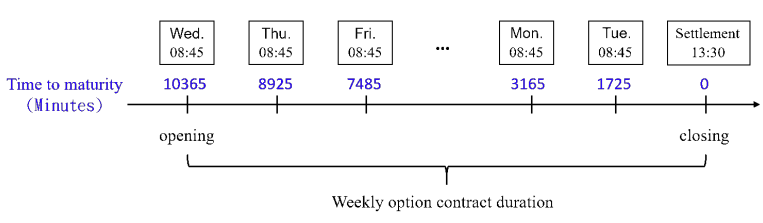

Fig. $11 T T M$ of the weekly option contract

We can measure whether the strategy is profitable based on the statistical heatmap of win-lose ratio. Take Figure 10 as an example, the $S L R$ of this heatmap is set to 1 , which means that the stop-loss will be performed immediately when the premium $P$ doubles. Apply the expected value formula can calculate that when the odds is 1 , the win-rate must be greater than 50 to be profitable. Therefore, the heatmap in this experiment will have a corresponding bottom line of win-lose ratio according to the $S L R$. If the $S L R$ is 1 , the bottom line of the win-lose ratio will be set to 0.50 . If the win-lose ratio is lower than 0.50 , the color in the heatmap will appear white (unprofitable); on the contrary, if the winlose ratio is higher than 0.50 , it will appear gradually red according to the win-rate. From an overall perspective in Figure 10, short put has a significantly higher win-lose ratio than short call.

Since financial transactions have the characteristics of time series, it represents that transaction data is continuous and time-sorted random variables. The frequency in this study is in minutes, but the time interval of high-frequency futures trading is even in seconds. In order to maintain the characteristics of time continuity in data, we further apply the concepts of convolution and smoothing. Think of the heatmap as a small square, and each small square represents the win-rate under the conditions of a specific PI and TTM. In order to reduce the gap between adjacent win-lose ratios, we can imagine the heatmap as a lot of nine square grids, and focus on the center point. Add all the adjacent grids and divide by 9 to get the average value which is the convolution win-lose ratio of the strategy. For example, as shown in the red dashed box in Figure 12, the upper and lower parts of the figure are the "win-rate of statistical methods" and "the win-rate after convolution" respectively. Let's take the red box nine square grid in the statistical method table as an example. The original win-lose-ratio at the center point (red circle) is 0.42. By adding up the win-lose ratio in the nine square grid (red dotted box), dividing by the number and taking the average which is the win-lose ratio of 0.40 after convolution. As a result, as shown in Figure 13, the top half and bottom half of the figure are the heatmap of win-lose ratio for short call and short put respectively. The vertical axis and horizontal axis of the graph are also the PI and TTM. After the win-lose ratio of the heatmap is averaged by convolution, the overall winlose ratio will become more concentrated and smoother which helps to select the trading range with a higher win-rate and stable.

The convolution method not only makes it easier for us to select the trading range with high win-lose ratio, but also avoids the problem of squeezing out the win-lose ratio by cutting the continuity feature value into segments. As shown in Figure 12, the upper and lower parts of the figure are the "win-rate of statistical methods" and "the winning rate after convolution" respectively. From the Statistical Method, we can see that the win-lose ratio of the block with the TTM between 1265 and 1261 and the $P I$ between 35 and 40 points (the green border) is about 0.62 . The win- 


\begin{tabular}{|c|c|c|c|c|c|c|c|c|}
\hline \multicolumn{9}{|c|}{ Win-rate of statistical methods } \\
\hline Premium $T$ TM & 1265 & 1264 & 1263 & 1262 & 1261 & 1260 & 1259 & 1258 \\
\hline$(45,50]$ & 0.33 & 0.29 & 0.29 & 0.29 & 0.35 & 0.35 & 0.36 & 0.33 \\
\hline$(40,45]$ & 0.25 & 0.35 & 0.36 & 0.36 & 0.32 & 0.38 & 0.42 & 0.39 \\
\hline$(35,40]$ & 0.67 & 0.6 & 0.62 & 0.62 & 0.62 & 0.48 & 0.46 & 0.47 \\
\hline$(30,35]$ & 0.48 & 0.47 & 0.4 & 0.43 & 0.42 & 0.59 & 0.53 & 0.45 \\
\hline$(25,30]$ & 0.33 & 0.41 & 0.4 & 0.37 & 0.41 & 0.37 & 0.35 & 0.45 \\
\hline$(20,25]$ & 0.54 & 0.59 & 0.59 & 0.62 & 0.59 & 0.65 & 0.67 & 0.62 \\
\hline$(15,20]$ & 0.71 & 0.71 & 0.74 & 0.71 & 0.71 & 0.69 & 0.69 & 0.67 \\
\hline$(10,15]$ & 0.59 & 0.61 & 0.53 & 0.46 & 0.51 & 0.52 & 0.54 & 0.63 \\
\hline$(05,10]$ & 0.66 & 0.68 & 0.7 & 0.67 & 0.71 & 0.69 & 0.7 & 0.67 \\
\hline \multicolumn{9}{|c|}{ Win-rate after convolution } \\
\hline Premium $T M$ & 1265 & 1264 & 1263 & 1262 & 1261 & 1260 & 1259 & 1258 \\
\hline$(45,50]$ & 0.32 & 0.31 & 0.32 & 0.33 & 0.34 & 0.36 & 0.37 & 0.36 \\
\hline$(40,45]$ & 0.43 & 0.42 & 0.42 & 0.43 & 0.42 & 0.42 & 0.40 & 0.40 \\
\hline$(35,40]$ & 0.47 & 0.47 & 0.47 & 0.46 & 0.47 & 0.47 & 0.46 & 0.44 \\
\hline$(30,35]$ & 0.49 & 0.49 & 0.48 & 0.48 & 0.48 & 0.47 & 0.46 & 0.46 \\
\hline$(25,30]$ & 0.47 & 0.47 & 0.48 & 0.47 & 0.49 & 0.51 & 0.52 & 0.51 \\
\hline$(20,25]$ & 0.54 & 0.56 & 0.57 & 0.57 & 0.57 & 0.57 & 0.57 & 0.57 \\
\hline$(15,20]$ & 0.62 & 0.62 & 0.62 & 0.61 & 0.61 & 0.62 & 0.63 & 0.63 \\
\hline$(10,15]$ & 0.65 & 0.66 & 0.65 & 0.64 & 0.63 & 0.64 & 0.64 & 0.65 \\
\hline$(05,10]$ & 0.63 & 0.63 & 0.61 & 0.60 & 0.59 & 0.61 & 0.63 & 0.64 \\
\hline
\end{tabular}

Fig. 12 Comparison of Win-lose ratio between Statistical and Convolution

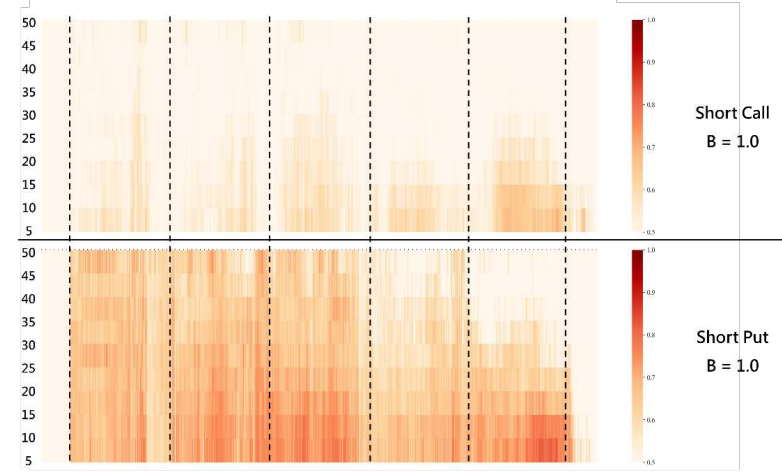

Fig. 13 Heatmap after convolution $(S L R=1.0)$

lose ratio of this block is greater than the expected value of 0.5 , which means it is profitable. However, the surrounding win-rate is relatively low. As can be seen from the "Win-rate after convolution", the win-rate after smoothing through convolution is about 0.47 (less than the expected value of 0.5 ). If we don't apply the convolution method are likely to mistakenly choose an unstable interval to trade, if there is a slight change in market conditions is easy to incur losses. In addition, it is also likely to miss the relatively stable trading range. As shown in Figure 12, the TTM from the "statistical methods win-lose ratio" is between 1263 and 1259 and the $P I$ is between 10 and 15 points (the blue border). A small part of this block has a win-lose ratio less than the expected value of 0.5 , so it is easy to be eliminated. However, because the win-rate around is relatively high which can be seen from the "win-lose ratio after convolution" that the win-rate obtained through the convo- lution method is about 0.64 (greater than the expected value of 0.5 ), which means that this block is stable and profitable. Therefore, the convolution method can avoid the problem of squeezing out the win-lose ratio, and prevent the wrong selection of a high win-lose ratio but unstable trading range, and then find a truly profitable range.

From the above, we have obtained a heatmap with a $S L R$ of 1 . We also tried several heatmaps with different $S L R$, including $S L R=0.5,1.0,1.5,2.0$. The different $S L R$ will also be matched with their corresponding bottom line of win-lose ratio. After calculating the expected value formula, the bottom line of win-lose ratio is $0.33,0.50,0.60$, and 0.66 respectively. As shown in Figure 14, the higher the $S L R$ setting $(S L R=2.0)$, the fewer red dots will be seen (the lower the win-rate), and the win-rate distribution of the heatmap with $\mathrm{B}=0.5$ is too even. Therefore, the study will use the $S L R$ as 1 for subsequent experiments.

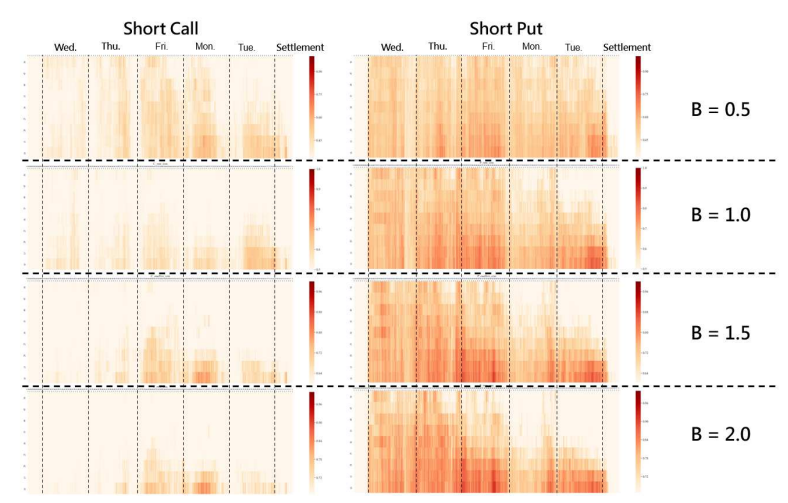

Fig. 14 Comparison of win-lose ratio with different $S L R$

We divided the historical data into training set and testing set. First, we use the training set (from June 2017 to December 2019) to calculate the win-lose ratio, and then use the testing set (from January 2020 to December 2020) to verify the overall effectiveness of the statistical model. As shown in Figure 15, the left half and the right half are the heatmap of the training set and the testing set respectively, while the upper and lower half are Short Call and Short Put respectively. This graph still shows that win-lose ratio of Short Put is much higher than Short Call. Since the risk management is also an important issue to our study, both Short Put and Short Call must be traded at the same time. It is not possible to trade Short Put only because of the high win-lose ratio of Short Put. The two are complementary to each other, it can reduce the risk of large losses. In order to be able to trade both call and put op- 
tions at the same time, it is necessary to choose a high and stable time interval. Therefore, we choose Monday, Tuesday, and settlement Wednesday for follow-up experiment and avoid the problem of uncertainty across the weekend.

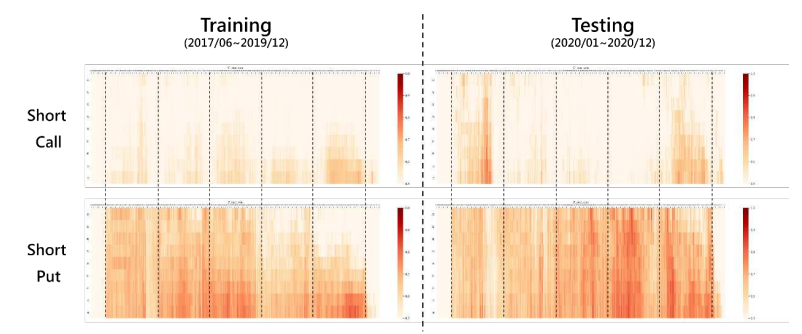

Fig. 15 Comparison of win-lose ratio with different dataset

In order to prove that the strategy performance is applicable, we compare the win-lose ratio of training set and testing set and measure whether the strategy meets expectations. As shown in Figure 16, this figure summarizes the win-lose ratio comparison between training set and testing set in different $P I$. The top layer and the bottom layer of the graph is $P I$ with premium of 45 to 50 points and $P I$ with premium of 5 to 10 points, and the left and right half of the graph are Short Call and Short Put respectively. The blue polyline and orange polyline are the win-lose ratio of training set and testing set. We can see from the figure that most of the training set and testing set win-lose ratio are quite close. On Tuesday, the win-lose ratio comparison (red box) with the $P I$ below 30 points, the win-lose ratio comparison is very close and higher than other weeks. The result of the comparison of the win-lose ratio in this figure can show that the trading strategy proposed by this research is stable and feasible.

4.2 Using random forest algorithm to predict the win-rate

This section will present the win-rate prediction of the random forest algorithm. First, due to the win-rate $W R$ of the trading strategy proposed in this study is defined as the number of times the premium is "not doubled" among all the number of transactions. Therefore, we set the "Whether to double" column in the data set as the classification target of the model. And use PI, TTM and Volatility as the input features of the model. In order to experiment with the impact of different fluctuation, we can subdivide the Volatility into the first 1

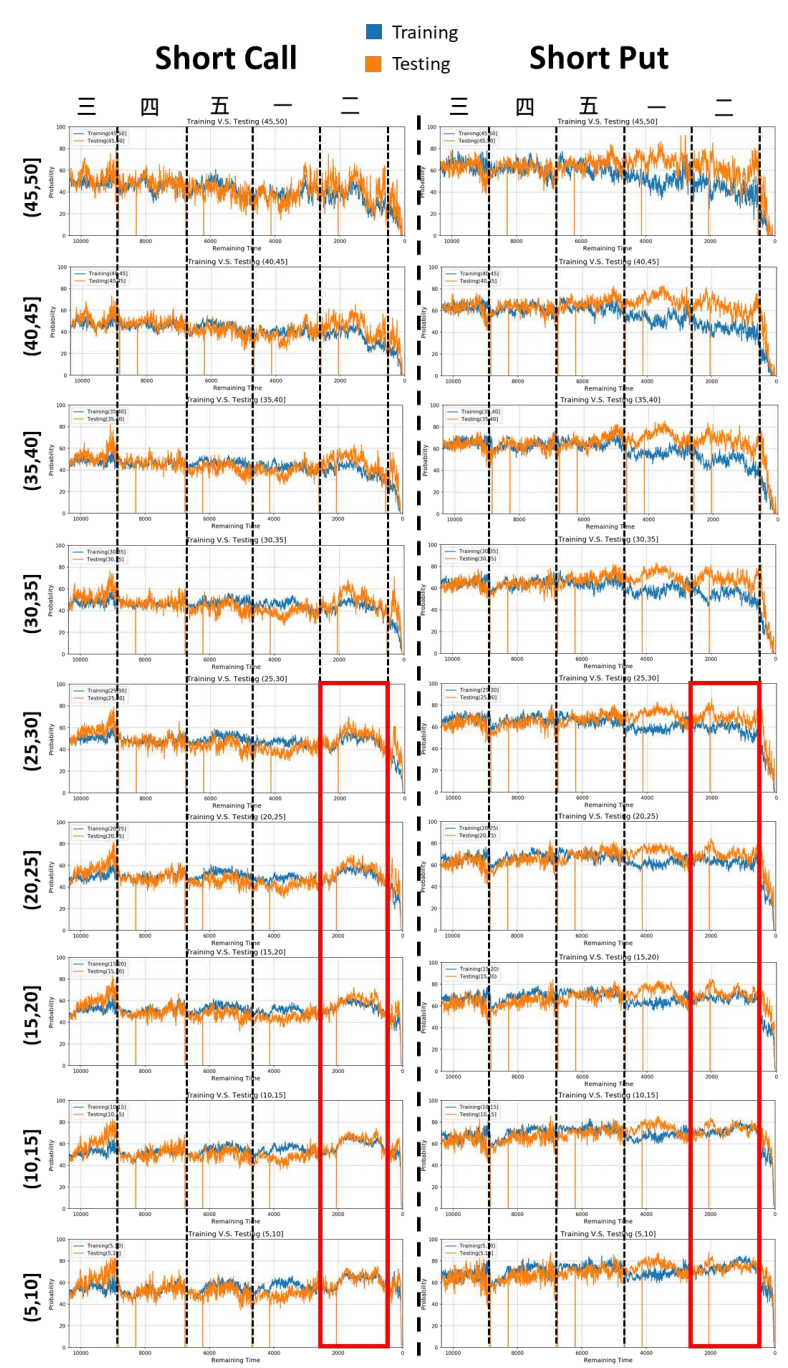

Fig. 16 Comparison of win-lose ratio with different dataset

minute, 3 minutes, 5 minutes, 15 minutes, 30 minutes, and the first 60 minutes.

Next, in order to evaluate the effectiveness of the model training, we divided the overall data set (data period from June 2017 to December 2020) in a time series into $70 \%$ for training set and $30 \%$ for testing set. This section will mainly focus on "Monday, Tuesday, Settlement Wednesday" to predict the $W R$ with the random forest model. Before putting the data into the model, we will first cut every 60 minutes into a segment based on the TTM. As shown in Figure 17, take Tuesday as an example. From the opening of the futures day trading at 08:45 in the morning until the closing of the night futures trading at 05:00 in the morning of the next day, the Time to Maturity can be divided into 21 parts for the trading period of Tuesday every 60 minutes as a segment. And then put each part of data into 
the model for training, and finally a total of 21 random forests can be produced based on the data of each time period. The reason for this approach is that $T T M$ is a key impact feature of this study. If all the TTM is put into the model for training at one time, the output of the experimental results may be too scattered and the influence of the TTM may decrease. Therefore, dividing the TTM into small segments according to the number of minutes can make the reference points of each random forest the same and independent and retain the value of the Time to Maturity.

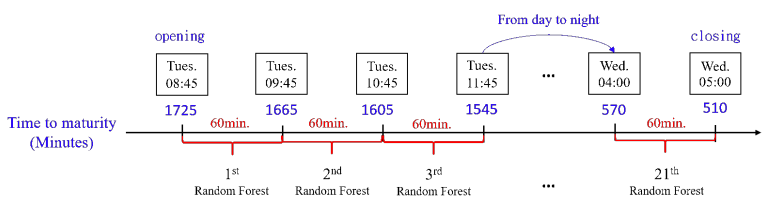

Fig. 17 Time to Maturity is divided into a random forest every 60 minutes

When the model training is completed, the test data will be used for verification. The win-rate represents the total number of times that each transaction data is extracted, how many times are estimated to be "undoubled" times. Then, we will merge each of its own random forests and use the heatmap to present the experimental results to see the overall win-rate of each week. As shown in Table 3, the heatmap of the winrate predicted by the random forest model on "Monday, Tuesday and Wednesday (settlement)". The left and right sides of the table are the results of Call and Put respectively, and the vertical axis of each heatmap in the table is $P I$, the horizontal axis from left to right is the $T T M$ from the opening time of the futures day trading at 08:45 am to the closing time of the futures night trading at 05:00 am. Only the TTM of Wednesday (settlement) only includes the day trading and only counts until 13:30 at the settlement time. The win-rate presented from the heatmap represents the result of the multi-layer judgment made by the algorithm using various eigenvalues and the sum of the results. These results indicate the number of times that each transaction data has been classified as "undoubled" in all forecast categories (including the forecasts as "not doubled" and "doubled"). But in fact, we will only conduct transactions on the parts that are "not doubled", and explore the proportion of "actually not doubled" in these transactions. This ratio can represent the probability of a real profit. Therefore, the heatmap in Table 3 can only show the prediction results of the random forest model, but this result does not fully represent the winrate (profitable probability) we really require.
Using Confusion Matrix allows us to further clarify whether the results predicted by the random forest model are consistent with actual data, and matrix data can help us calculate the probability of real profitability. Table 4 summarizes the confusion matrix predicted by the random forest algorithm in this study. The rows and columns of the confusion matrix represent the predicted value and the actual value, respectively. Taking this research as an example, we will observe whether the option premium will double before the contract is settled. From the standpoint of the option seller, it is beneficial to the seller that the premium has not doubled. Therefore, if the actual value of the premium is "not doubled", it will be marked as 1 , and if the premium is "doubled", it will be marked as 0 . The result of random forest prediction is the predicted value. If the predicted result is that the premium is "not doubled", it is classified as 1, and we should enter the market; on the contrary, if the predicted result is that the premium is "doubled", it is classified Is 0 , and we should not take trading actions.

As shown in Table 4, we have consolidated the confusion matrix for Monday, Tuesday, and settlement Wednesday. The percentages of correct predictions for Call and Put on "Monday" are $54 \%$ and $62 \%$, respectively, indicating that 50 to $60 \%$ of the predictions are accurate. On the other hand, "Tuesday" Call and Put accounted for $59 \%$ and $68 \%$ of the correctness predictions respectively, indicating that about $60 \%$ and $70 \%$ of the predictions were accurate. Finally, the correctness forecasts of "Wednesday (Settlement)" Call and Put accounted for $71 \%$ and $70 \%$ respectively, indicating that $70 \%$ of the forecasts were accurate.

But compared to the accuracy of model prediction, what makes us want to know is the precision of model prediction. Precision refers to the ratio of correct predictions among all the results predicted by the model, that is, the degree of similarity between the predicted value of the model and the actual reference value. And "precision" refers to the proportion of the model predicted to be positive and the actual value is also positive, which is equivalent to the degree to which the predicted value matches the actual value within the specified range of conditions. Taking this study as an example, when the random forest model predicts that the premium classified as positive is "not doubled", we should make a transaction. When the model predicts that the premium classified as negative is "doubled", we will not take any action. It means that when the classification result is negative, there will be neither loss nor profit for the trading strategy. Therefore, we only need to focus on the classification results predicted by the model to be positive. In other words, since we 
Table 3 Random Forest Win-Rate Estimated Heatmap Integration Table
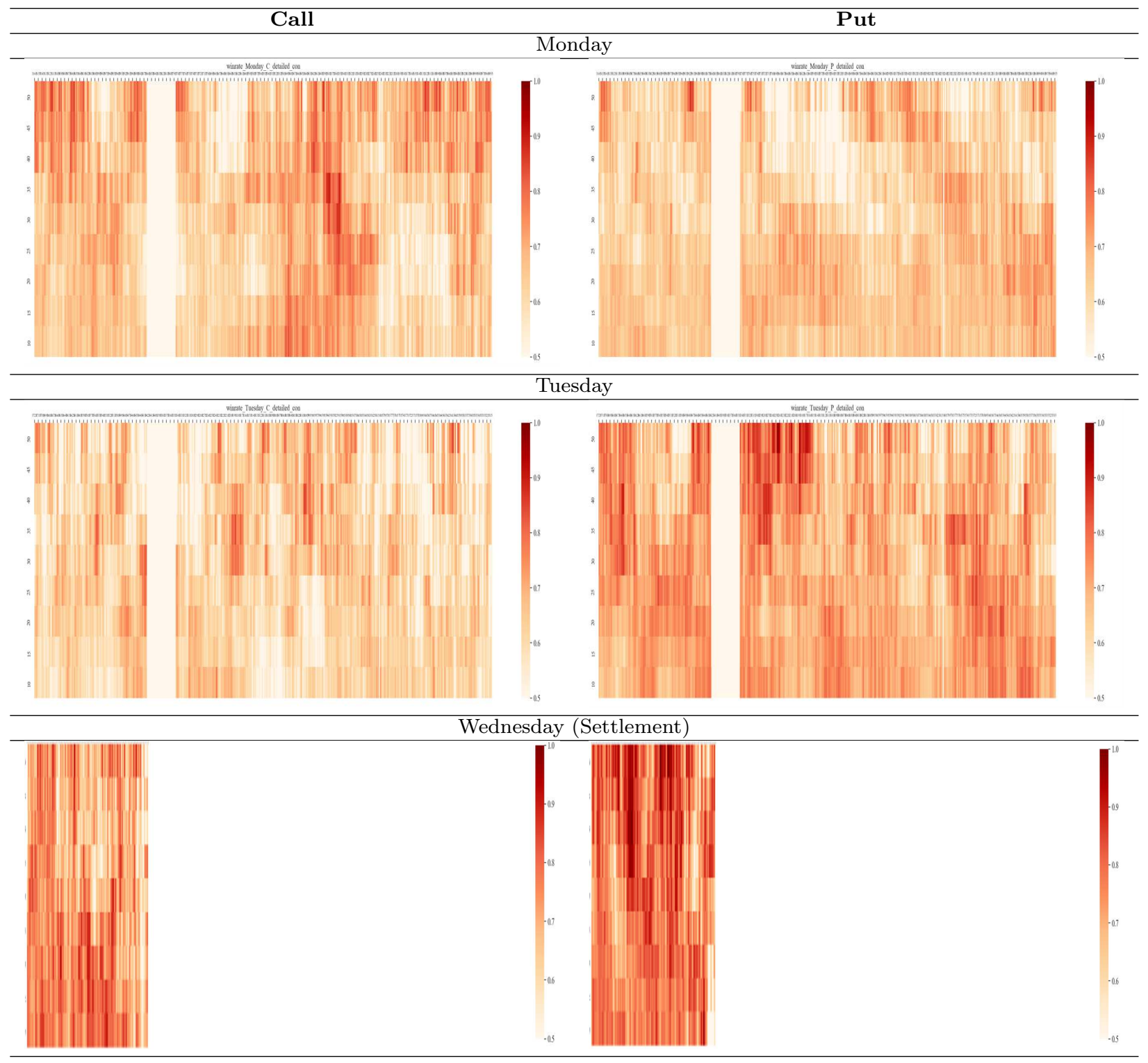

only trade when the classification result predicted by the model is "not doubled" the premium, we only need to focus on the proportion of these positive predicted values and the actual value is "not doubled". Therefore, precision is equivalent to the probability that the trading strategy may be profitable, which is the win-rate we really want to know.

Table 5 is the precision calculated by the classification results of random forest prediction in this study. Each random forest will eventually get a precision ratio, and when the ratio exceeds $50 \%$, it means that the strategy can be profitable. In the following table, we have compiled the precision ratios of each random forest on Monday, Tuesday, and settlement Wednesday, and presented it as a histogram. The light red and light purple in the histogram represent Call and Put, respectively. The horizontal axis in the figure represents each random forest (the Time to Maturity is 60 minutes as an interval). The vertical axis is the precision of random forests. The maximum is $1(100 \%)$ and the minimum is $0(0 \%)$. First, from "Monday" we can see that the precision ratios of the Put are all greater than $50 \%$ (the red dotted line) and the average is about 0.75 , which means that when we follow the classification results of random forest predictions When trading, there is a $75 \%$ chance that a profit can be made. However, the precision of the Monday Call is relatively unsatisfactory. Most of the precision do not exceed $50 \%$, and there is a risk of 
Table 4 Confusion matrix unified table predicted by random forest algorithm

\begin{tabular}{|c|c|c|c|c|}
\hline \multirow{3}{*}{ Weekday } & \multicolumn{4}{|c|}{ Call Put } \\
\hline & \multirow{2}{*}{\multicolumn{2}{|c|}{ Confusion Matrix }} & \multicolumn{2}{|c|}{ Actual Value } \\
\hline & & & Not Doubled & Doubled \\
\hline \multirow[b]{2}{*}{ Mon. } & \multirow[b]{2}{*}{ Predicted Value } & Not Doubled & $235943(48 \%) \quad 39431$ & $184366(37 \%) \quad 201$ \\
\hline & & Doubled & $42777(9 \%)$ & $31132(6 \%) \quad 23210(3 \%)$ \\
\hline \multirow{2}{*}{ Tues. } & \multirow{2}{*}{ Predicted Value } & Not Doubled & $239682(54 \%)$ & $146777(33 \%)$ \\
\hline & & Doubled & $36842(8 \%)$ & $22921(5 \%)$ \\
\hline \multirow{2}{*}{ Wed. (Settlement) } & \multirow{2}{*}{ Predicted Value } & Not Doubled & $49730(70 \%)$ & $17505(25 \%)$ \\
\hline & & Doubled & $3272(5 \%)$ & $877(1 \%)$ \\
\hline
\end{tabular}

loss. From the data of "Tuesday", it can be found that most of the precision ratios of call rights and put rights are more than $50 \%$, and the average values are 0.59 and 0.77. Finally, from the histogram of "Settlement Wednesday", we can see that the precision of Call and Put are both more than $50 \%$ and the average values are 0.70 and 0.74 , respectively, indicating that there is a high probability of about $70 \%$ of profit. In summary, the prediction precision ratio of most random forest algorithms is greater than $50 \%$, and the precision of put options can be as high as $75 \%$ on average. Among them, the precision data of "Settlement Wednesday" is the most ideal.

From precision data, we can know the probability of a profitable trading strategy. In the process of constructing the random forest model, the feature selection and classification basis of each decision tree in it are also important influencing factors of the algorithm. The characteristic values used in the random forest model of this study include: Premium, TTM and Volatility in different frequency, including one minutes, three minutes, five minutes, fifteen minutes, thirty minutes and sixty minutes.

Table 6 unifies Monday, Tuesday, and settlement Wednesday, and the importance of each feature value changes with TTM. Each table contains a total of 3 graphs, the first and second are the feature importance line graphs of Call and Put respectively. The vertical axis of the line graph represents the importance of features, the higher the value, the higher the importance; the horizontal axis represents each random forest. In the line chart, different line colors respectively represent a characteristic value, and there are a total of 8 different characteristic values. The third graph in each table is a bar graph of the feature importance of Call and Put. The vertical axis represents the value of each feature, the horizontal axis represents the importance of the feature. The light red and the light purple in the horizontal bar graph represents Call and Put respectively. First, from the line and bar charts of "Monday" and "Tuesday", whether it is a call or a put, the influence of "60-minute volatility" ranks first, followed by "30-Minute volatility" and "15-minute volatility", volatility is a very important feature. The longer the volatility time period, the deeper the impact on model classification. In addition, from the line chart and bar chart of "Settlement Wednesday", it can be found that the importance of the feature of " Premium" greatly exceeds other feature values. Time value has a huge impact on option sell-side. Option sell-side can earn time value through the passage of time. The time value of "Settlement Wednesday" for the option buyer has dropped rapidly. When the option premium approaches zero at the time of settlement, the seller gains. Therefore, as the experimental results show, it is reasonable to interpret that the importance of the feature of "Premium" in the random forest model can greatly surpass other feature values on "Settlement Wednesday".

\subsection{Comparison of precision between statistical} methods and random forest algorithm

Table 7 is a table of the precision comparison between statistical methods and random forest prediction. The left half and right half of the table are respectively the statistical model probability to bring $50 \%$ and $60 \%$ precision line charts. The vertical axis of the line graph is the precision, the maximum value is 1 (100\%), and the minimum value is $0(0 \%)$; the horizontal axis is each random forest. The color of dark red and dark purple represents the Call and Put of the "statistical method" respectively, while the light red and light purple represent the Call and Put of the "random forest algorithm". First, from the line chart of "Monday" and "Tuesday", 
Table 5 Random Forest prediction precision table

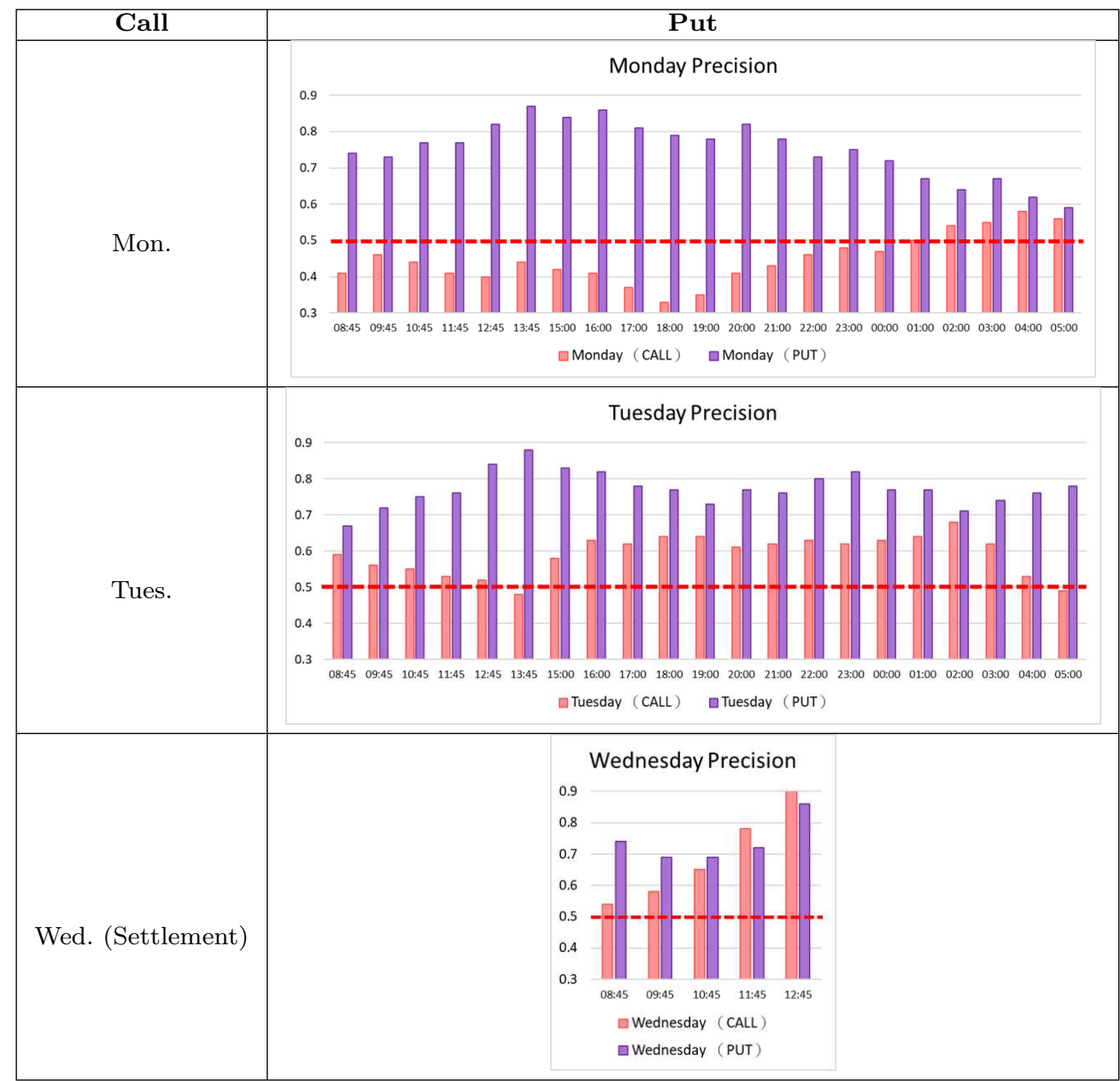

the precision of the statistical method and the random forest algorithm are roughly the same, and it can be found that the precision of Put is significantly higher than that of Call. In addition, Call and Put are complementary, whenever one of them has an increased precision, the other side will decrease which can reduce the risk of loss. Then, from the line chart of "Settlement Wednesday" at the beginning, the precision of the statistical method and the random forest algorithm is almost the same, but when it is close to the settlement, the precision of the random forest greatly surpasses the statistical method. It means that when we do transactions according to the classification prediction of the random forest, the profit opportunity on settlement Wednesday is greatly exceeded by the statistical method, and the precision of the random forest algorithm can be as high as about $90 \%$ when it is close to the settlement.

\section{Conclusions}

This paper is devoted to the research and development of profitable option sell-side trading strategies, and proposes an operating mechanism for stop-loss. In addition, statistical methods and random forest algorithms are used to estimate the win-rate of the strategy. The win-rate represents the proportion of all transactions that the premium has not doubled before settlement, and we can also express it with precision. In the experimental results of statistical methods, we found through the heatmap that the win-rate of Short Put is significantly higher than that of Short Call. In order to explore the accuracy of win-rate estimation, we divided the data into training set and testing set. The data results show that the win-rate of the training set and the testing set are quite close. Among them, the trading range on Tuesday is the most ideal, and the win-rate can be as high as about $70 \%$. In addition, in the experimental results of the random forest algorithm, through the 
Table 6 The importance of features in random forest prediction

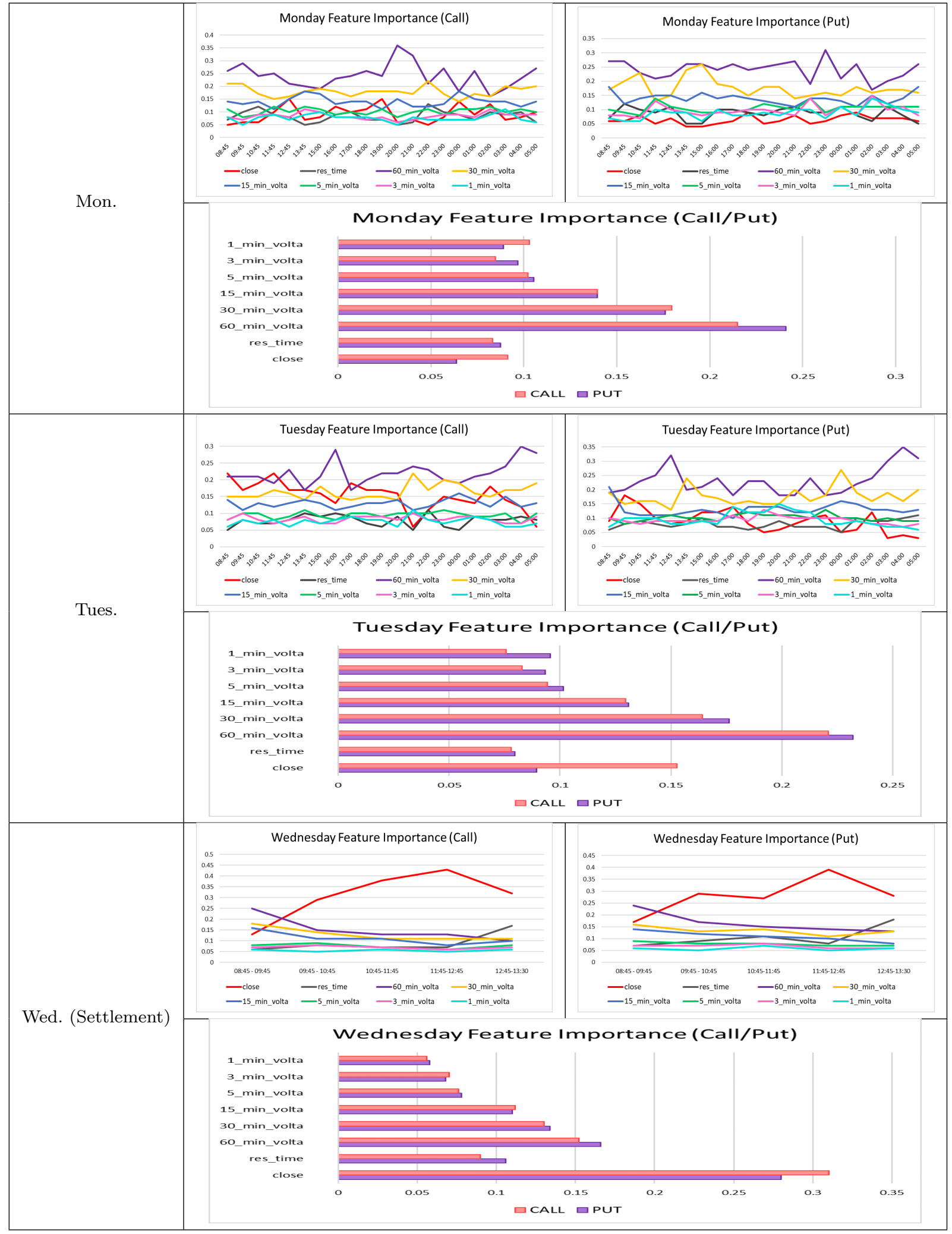


Table 7 Comparison of precision between statistical methods and random forest predictions

\begin{tabular}{|c|c|c|}
\hline Weekday & Probability Threshold $=50 \%$ & Probability Threshold $=60 \%$ \\
\hline Mon. & 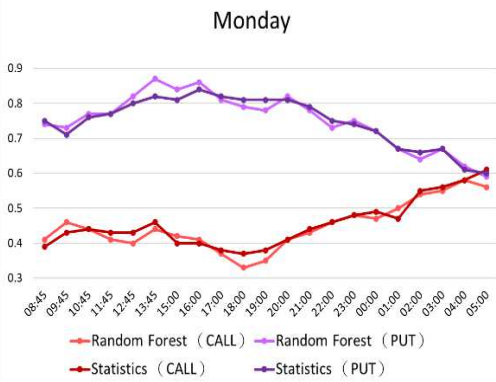 & $\begin{array}{l}0.8 \\
\rightarrow \text { - Standistics (CALI) } \rightarrow \text { Statistics (PUT) }\end{array}$ \\
\hline Tues. & $\begin{array}{l}0.4 \\
\rightarrow \text { - Random Forest }(\text { CALL) } \rightarrow \text {-Random Forest (PUT) } \\
\rightarrow \text { Statistics (CALL) } \rightarrow \text {-Statistics (PUT) }\end{array}$ & $\begin{array}{l}0.8 \text { - Tuesday } \\
\rightarrow \text {-Statistics (CALL) } \rightarrow \text { Statistics (PUT) }\end{array}$ \\
\hline Wed. (Settlement) & 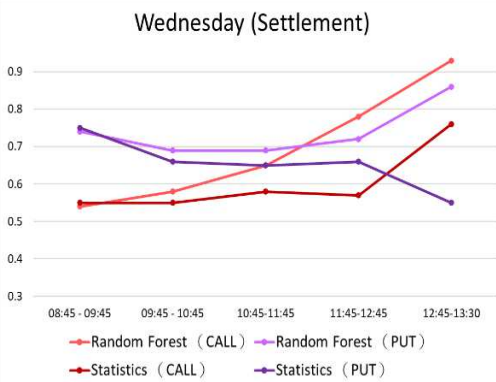 & 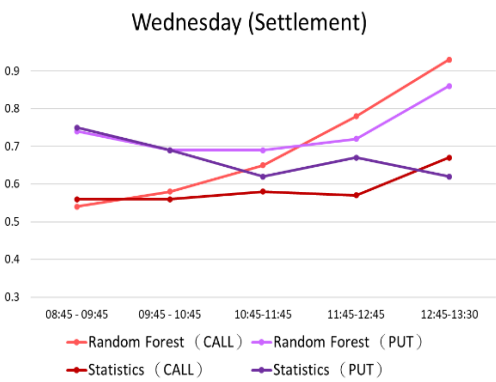 \\
\hline
\end{tabular}

classification prediction of the model, we found that the forecast precision of settlement Wednesday is very satisfactory. Both the Call and Put can reach $75 \%$. When approaching the settlement, the prediction accuracy of Random Forest can reach nearly 90\%, which greatly surpasses statistical methods. The experimental results can confirm that the trading strategy proposed by this paper can effectively achieve risk control through the development of a stop-loss mechanism with a fixed premium double multiple. And apply statistical methods and random forest algorithm to estimate the win-rate of the strategy, and screen out the trading range with higher profit and stable. The precision predicted by the model classification can prove that the strategy is practical and profitable. In future, we can build models through more ways, such as neural networks and financial engineering, and add more different features for model training. In addition, after we have screened out profitable trading ranges, we can simulate investment funds and use historical data to do back-testing and explore the actual profit and loss value. Finally, it is expected that the option sell-side trading strategy proposed in this paper can achieve the desired effect of long-term stable returns.

Acknowledgements This work was supported in part by Ministry of Science and Technology, R.O.C under grant number MOST 109-2221-E-027 -106 -

\section{Declaration}

Conflict of interest

The authors declare that they have no conflict of interest.

Ethical approval

This article doesn't contain any studies with human participants or animals performed by any of authors. 


\section{References}

Belgiu M, Drăgut L (2016) Random forest in remote sensing: A review of applications and future directions. ISPRS journal of photogrammetry and remote sensing 114:24-31

Bermin HP, Holm M (2021) Kelly trading and option pricing. Journal of Futures Markets 41(7):987-1006

Bermin HP, Holm M, et al. (2019) Kelly Trading and Market Equilibrium. Lund University, School of Economics and Management

Breiman L (2001) Random forests. Machine learning 45(1):5-32

Brenner M, Subrahmanyam MG (1994) A simple approach to option valuation and hedging in the blackscholes model. Financial Analysts Journal 50(2):2528

Cox JC, Ross SA, Rubinstein M (1979) Option pricing: A simplified approach. Journal of financial Economics 7(3):229-263

Evans RB, Geczy CC, Musto DK, Reed AV (2009) Failure is an option: Impediments to short selling and options prices. The Review of Financial Studies 22(5):1955-1980

Jang JH, Yoon J, Kim J, Gu J, Kim HY (2021) Deepoption: A novel option pricing framework based on deep learning with fused distilled data from multiple parametric methods. Information Fusion 70:43-59

Kelly Jr JL (2011) A new interpretation of information rate. In: The Kelly capital growth investment criterion: theory and practice, World Scientific, pp 25-34

Liu D, Liang Y, Zhang L, Lung P, Ullah R (2021) Implied volatility forecast and option trading strategy. International Review of Economics \& Finance 71:943-954

MacLean LC, Thorp EO, Ziemba WT (2010) Good and bad properties of the kelly criterion. Risk 20(2):1

MacLean LC, Thorp EO, Ziemba WT (2011) The Kelly capital growth investment criterion: Theory and practice, vol 3. world scientific

Merton RC (1973) Theory of rational option pricing. The Bell Journal of economics and management science pp 141-183

Nabipour M, Nayyeri P, Jabani H, Shahab S, Mosavi A (2020) Predicting stock market trends using machine learning and deep learning algorithms via continuous and binary data; a comparative analysis. IEEE Access 8:150199-150212

Pal M (2005) Random forest classifier for remote sensing classification. International journal of remote sensing 26(1):217-222

Ruf J, Wang W (2020) Neural networks for option pricing and hedging: a literature review. Journal of Com- putational Finance, Forthcoming

Stutzer M (2011) On growth-optimality vs. security against underperformance. In: The Kelly capital growth investment criterion: theory and practice, World Scientific, pp 641-653

Thorp EO (2011) The kelly criterion in blackjack sports betting, and the stock market. In: The Kelly capital growth investment criterion: theory and practice, World Scientific, pp 789-832

Wiggins JB (1987) Option values under stochastic volatility: Theory and empirical estimates. Journal of financial economics 19(2):351-372

Wu ME, Chung WH (2018) A novel approach of option portfolio construction using the kelly criterion. IEEE Access 6:53044-53052

Wu ME, Hung PJ (2018) A framework of option buyside strategy with simple index futures trading based on kelly criterion. In: 2018 5th international conference on behavioral, economic, and socio-cultural computing (BESC), IEEE, pp 210-212

Wu ME, Tsai HH, Tso R, Weng CY (2015) An adaptive kelly betting strategy for finite repeated games. In: International conference on genetic and evolutionary computing, Springer, pp 39-46

Wu ME, Wang CH, Chung WH (2017) Using trading mechanisms to investigate large futures data and their implications to market trends. Soft Computing 21(11):2821-2834

Yang H, Choi HS, Ryu D (2017) Option market characteristics and price monotonicity violations. Journal of Futures Markets 37(5):473-498 